\title{
Looking Towards the Future of Strain Scanning Using Neutron Diffraction
}

\author{
R.C.Wimpory ${ }^{1, a^{*}}$ and M. Boin ${ }^{1, b}$ \\ ${ }^{1}$ Helmholtz-Zentrum Berlin für Materialien und Energie, \\ Hahn-Meitner-Platz 1, D-14109 Berlin, Germany \\ arobert.wimpory@helmholtz-berlin.de, bboin@helmholtz-berlin.de
}

\begin{abstract}
Keywords: instrument control, data acquisition, simulation, dead time reduction, proposal submission, Finite Element Modelling [FEM]
\end{abstract}

\begin{abstract}
All aspects of instrument control, data acquisition, simulation and analysis are expected to merge in the future. For instance real time data analysis will feed back influencing the instrument control in order to optimize the measurement time and simulations themselves will control the instrument. This presentation will discuss how the all-important pre-planning of a measurement can be optimized and used to define the whole measurement, efficiently and effectively using the neutron beamtime.
\end{abstract}

\section{Introduction}

The natural tendency for experimentation is the need to do things more quickly, more accurately, more effectively and a need to know exactly what happened at each step retrospectively (especially if something happens that cannot be easily explained). This is especially true when it comes to neutron beam time, which is precious due to the limited number of neutron sources and limited chances of getting beam time in the first place. Similar concepts of course also apply to other measurement methods such as with synchrotron radiation but the authors will use the example of neutron radiation here.

Arguably the most important part of an experiment is the pre-planning. Neutron beam time is expensive and so there is a need to fully optimize experiments before any neutrons are used. This also ensures that any potential problems are dealt with well in advance therefore maximizing a measurements chance of success.

It only takes a little bit of imagination to see in which direction things will go and the ideas and concepts expressed and suggested here are not far beyond what is technically possible and extend and build on current developments and the current Zeitgeist.

\section{Interactive proposal submission}

The pre-planning of a measurement can start with the neutron beam time proposal itself. There is no reason why in the future a new generation of 'smart proposal submission' could not be used when applying for neutron beam time, making the whole process more interactive. A new way of submitting a proposal could be used to simulate the experiment for the particular instrument, optimizing the experiment and proposal and the output could also be used to create the final experiment protocol (input file of the measurement) if the proposal is accepted. Feedback to the submitter could take the form of an animated CAD movie show all stages of the simulated proposed 
experiment. This would be especially useful for people new to the technique and one can see immediately the whole process. A number of things could be calculated and optimized already at this stage, such as gauge volume size (especially if predictions of strain gradients are already available), measurement time, reduction of neutron path lengths (e.g. with the aid of an Eulerian cradle), the most suitable Bragg reflections and the most suitable auxiliary equipment needed (for optimization of heating and cooling times for a furnace for example) etc.

The concept of optimizing an experiment off-line already exists with the SScanSS software development by J.A. James et al. [1] which provides a virtual experimental simulation. This software was developed for facilitating the planning of strain scanning experiments prior to arrival at the facility, and also automatically generates the instrument control sequence for use during the experiment. The sample is represented as a 3D outline model which is scanned using a laser or a coordinate measurement machine (usually at the facility where the neutron instrument is) which is imported into the software. The user can mark the positions of the measurement points with reference to this virtual sample and can specify which strain components are to be measured for each specific point. The SScanSS software generates, automatically, the required sample table movements for the measurement of the selected points, based on the coordinates of fiducial points measured at the start of the experiment.

One could already imagine what the future could be, building on those ideas such as those contained within the SScanSS software. Simulation software that encompasses a complete description of everything including the instrument's neutron optics and a realistic detailed sample representation could optimize itself and control the whole measurement interactively and this could be already initiated at the proposal submission stage. Presently Monte Carlo/Ray tracing programs such as McStas [2], VITESS [3] and RESTRAX [4] have the ability to simulate and optimize neutron instruments. Progress has already been made in making more complex virtual samples descriptions for neutron simulations [5].

Of course the more information one can input the better the simulation. Different degrees of complexity could be added and it is quite often for example that a component or sample has already been modelled (such as with a finite element model) and so this itself could be inputted. Programs such as ABAQUS [6] and ANSYS [7] are currently available for Finite Element Modelling (FEM). If a model does not yet exist, then simple inputted dimensions could be used for a sample of a simple geometry. If the specimen is of a more complicated geometry then a 3D scan maybe necessary. It is not entirely inconceivable that a 'phone app' or another solution could be made to scan sample shapes in the future rather than relying on expensive laser scanners or coordinate measurement machines which are normally housed at the neutron facility.

If the specimen geometry has not yet been specified, the simulation could also be used to design the best specimen geometry that will satisfy and optimize both measurement time and high-light the problem that needs to be solved from the investigation. This could in principle be combined with a finite element modelling program interacting with the simulation program.

\section{Smart instrument positioning, simulation and 'real-time archiving'}

The technology exists to constantly monitor the absolute and relative positions of all the components of an instrument accurately at all times (including the monochromator and optics). To achieve the required accuracy, this could be done using a mixture of techniques, distance sensors (e.g. lasers) with the aid of optical cameras with intelligent visual recognition, etc., a bit like a local GPS system. Smart sensors could also tell if a particular type of furnace, stress rig or baseplate is on the instrument, making the instrument more 'alive'. The whole history of the instrument could be 
constantly time-stamped and this would be important for constant 'real-time archiving' and the relevant information ending up in the final data file for a particular measurement. The ultimate target would be to be able to 'replay' the whole measurement with an accurate time line of an experiment including all acquired detector data and analysis. This could be done with the aid of a data format such as NeXus [8] which contains many more layers of information than are normally stored in a typical data file.

This constant availability of position and status data would be essential for simulations and collision avoidance. This information can be constantly available to smart automatic simulation routines that suggest the best configuration or automatically set the best configuration for a measurement, for instance the information that has already been acquired from the proposed 'interactive proposal submission' system. The optimized settings for a measurement can be selected and the instrument could be ready within moments. The accurate knowledge as to where each component is would facilitate automatic calibration and this would save beamtime in setting up the instrument each time as one could do this also in principle without the aid of neutrons.

Constant feedback between measurement and simulations should also be able to provide better estimations of uncertainty too. Actual gauge volume sizes (including neutron divergence) can be simulated, calculated and verified with the aid of, for instance, neutron cameras in the primary beam. This can also be important for things like grain size issues which often cause difficulties and compromise accurate strain estimation. A real-time simulation acting on data acquired from the detector could automatically interact with the measurement itself, for instance continuously oscillating a specimen to improve grain size statistics when needed. The simulation can also be used to correct possible aberrations in real-time, interacting with the data acquisition and data analysis. With a greater degree of sample knowledge and simulation, gauge volume reduction at strategic points to capture steep strain gradients could also be automated. Finite element models often use different sized modeling elements at different places depending on the expected strain gradients in a sample to speed up calculation of a simulation, an analogous approach could be done with neutrons.

Constant acquisition of data and constant simulation is also of importance for smart or selflearning simulations (for instance by genetic algorithms) that could constantly suggest ideas to improve the instrument, for instance trace back to find where unnecessary background is coming from. All possible uncertainties can be simulated in order to double check the final fitting errors and possibly discover unexpected sources of errors and aberrations.

If new developments from other developers around the world were immediately made available as simulation modules (in a standard way) this could allow for automatic simulations of an instrument to see if the new development could benefit the instrument. Also an open source nature of an instrument could also allow the user community to simulate and continuously suggest ideas.

\section{Real-time Diagnostics}

With several systems working closely together (such as simulation/instrument control and archiving) it will become easier to diagnose and predict problems that may arise. There is nothing worse than starting a measurement for it to stop a few hours after walking away from it. Mirko Boin has already implemented automatic SMS alerts to the instrument responsible and users (on the E3 residual stress diffractometer at the Helmholtz-Zentrum Berlin) in the case when the measurement stops for some unexpected reason. A natural extension would be an option for the measurement to automatically restart at the correct point (if the reason for stopping is without the need to repair a component). The position information could be recovered from the real-time archiving if necessary. 
Other diagnostic possibilities could be alerts to when the detector gas pressure become too low and profiling and analyzing motion and vibration of motors to see if there are any changes that may indicate possible future failure.

\section{Immediate data availability and intelligent publishing}

In the future the accessing and sharing of knowledge will certainly be more fluid and will be more and more immediate. A good piece of research will certainly have information that will be relevant for future generations. If all this data could be made available in a standard way, then all the current knowledge about the sample being measured (and the way it was made) could be made immediately available. This could take the form of automatic Bragg peak recognition/suggestion on the instrument, automatic access to material properties (including up-to-date elastic constants), intergranular strain information, expected strains and stresses from a particular weld technique, background knowledge about the influence of texture etc. Some of this information could in principle be already made available at the proposal submission stage to high-light as to why a measurement is new and needs to be carried out.

New accurate information must be available and not be hidden away to be discovered years later. This may involve some type of 'knowledge tabs' from all future publications so that the information is available for immediate automatic use for all applications.

\section{Output}

There is no reason why the final output file cannot be a complete 'movie' of everything that happened in the measurement. This could be similar to a type of animated CAD file, showing all the exact instrument positions used, detector data, and reactor power etc., all completely timestamped. The file can also contain all peak fits and the ability to easily output data in more manageable visualizations for presentations or papers. This could be visualized schematically and also with the possibility a real photographic overlay from the optical camera data, similar to web based layered maps, except more dynamic. This can be also ideal for teaching.

The whole process can easily be verified to see if it complied with any standard. Because the whole process is potentially more thorough and transparent, the need for standardization is even possibility redundant when it can be proved that the measurement has been carried out taking absolutely every source of uncertainty possible.

This information could be easily merged or compared with other measurements on the same or nominally the same specimen (such as in round robin exercises or complementary methods). For interrupted measurements having enough information could mean that the experiment could be continued on the same or different instrument at a later date from exactly the last position measured.

This could be based on an already existing file format such as NeXus [8]. NeXus is developed as an international standard by scientists and programmers and contains many more layers of information than are normally stored in a data file.

\section{Summary}

With ever-increasing computer power the concept of using simulations to augment or execute a measurement (involving a detailed description of the instrument and sample) becomes more realistic. Technology is moving along at an amazing pace and technology works best when systems are compatible with each other with automatic interfacing. All this can be taken advantage of in 
future. The future of instrument control could be a combination of different current ideas and concepts, such as SScanSS [1] for the basic experimental planning, a Monte Carlo/Ray-tracing program $[2,3,4,5]$ combined with a finite element program [5,6] to add a layer of more detailed knowledge about the instrument and sample. Together with an interaction with neutron detector, neutron camera information and exact knowledge of the instrument's components positions, an intelligent system would be able to optimize the strain measurement completely. The vast majority of the measurement could be optimized already at proposal submission stage using an 'Interactive proposal submission' system. The advantage of this would not only be the efficient use of beam time but also the confidence in the results obtained. With a more thorough description of the instrument and especially of the sample, many influencing factors for a measurement could be described and quantified.

Publishing is expected to become faster and the need to make knowledge available for immediate use will be invaluable and this can be seen helping with experimentation in the future. A final output file could be a form of animated CAD file with all aspects of the measurement. The ultimate target would be to be able to 'replay' the whole measurement with an accurate time line of an experiment including all acquired detector data and analysis, instrument positions and all other information acquired.

\section{References}

[1] J.A. James, J.R. Santisteban, L. Edwards and M.R. Daymond, 2004. A Virtual Laboratory for Neutron and Synchrotron Strain Scanning. Physica B: Condensed Matter, 350(1-3), pp. E743E746.

[2] K. Nielsen, K. Lefmann, Monte Carlo simulations of neutron-scattering instruments using McStas, Physica B: Condensed Matter, Volume 283, Issue 4, 3 June 2000, Pages 426-432, ISSN 0921-4526, DOI: 10.1016/S0921-4526(00)00381-1.

[3] G. Zsigmond, K. Lieutenant, S. Manoshin, H. N. Bordallo, J. D. M. Champion, J. Peters, et al., A survey of simulations of complex neutronic systems by VITESS, Nuclear Instruments and Methods in Physics Research Section A: Accelerators, Spectrometers, Detectors and Associated Equipment, Volume 529, Issues 1-3, Proceedings of the Joint Meeting of the International Conference on Neutron Optics (NOP2004) and the Third International Workshop on PositionSensitive Neutron Detectors (PSND2004), 21 August 2004, Pages 218-222, ISSN 0168-9002, DOI: 10.1016/j.nima.2004.04.205.

[4] J. Saroun, J. Kulda, RESTRAX -- a program for TAS resolution calculation and scan profile simulation, Physica B: Condensed Matter, Volumes 234-236, Proceedings of the First European Conference on Neutron Scattering, 2 June 1997, Pages 1102-1104, ISSN 0921-4526, DOI: 10.1016/S0921-4526(97)00037-9

[5] BOIN, M., et al., "Validation of Bragg edge experiments by Monte Carlo simulations for quantitative texture analysis", Journal of Applied Crystallography, 44 (2011) pp. 1040-1046.

[6] H.D. Hibbitt, ABAQUS/EPGEN--A general purpose finite element code with emphasis on nonlinear applications, Nuclear Engineering and Design, Volume 77, Issue 3, 2 February 1984, Pages 271-297, ISSN 0029-5493, DOI: 10.1016/0029-5493(84)90106-7.

[7] Stefan Reh, Jean-Daniel Beley, Siddhartha Mukherjee, Eng Hui Khor, Probabilistic finite element analysis using ANSYS, Structural Safety, Volume 28, Issues 1-2, Structural Reliability Software, January-April 2006, Pages 17-43, ISSN 0167-4730, DOI: 10.1016/j.strusafe.2005.03.010.

[8] http://www.nexusformat.org/ 